\title{
Generating functions for the number of permutations with limited displacement
}

\author{
Torleiv Kløve* \\ Department of Informatics, \\ University of Bergen, N-5020 Bergen, Norway \\ Torleiv.Klove@ii.uib.no
}

Submitted: Jan 13, 2009; Accepted: Aug 6, 2009; Published: Aug 14, 2009

Mathematics Subject Classifications: 05A15, 94B60

\begin{abstract}
Let $V(d, n)$ be the number of permutations $p$ of $\{1,2, \ldots, n\}$ that satisfy $\left|p_{i}-i\right| \leqslant$ $d$ for all $i$. Generating functions for $V(d, n)$, for fixed $d$, are given.
\end{abstract}

\section{Introduction.}

The problem considered in this paper is the enumeration of permutations which satisfy $\left|p_{i}-i\right| \leqslant d$ for all $i$. The motivation comes from coding theory. A permutation array is a set of permutations of $[n]=\{1,2, \ldots, n\}$. Recently, Jiang et al. [1,2] showed an application of permutation arrays to flash memories, where they used different distance metrics to investigate efficient rewriting schemes. In [4], we studied the multi-level flash memory model, using the Chebyshev metric.

More precisely, we consider the distance $d_{\max }$ between permutations defined by

$$
d_{\max }(p, q)=\max _{j}\left|p_{j}-q_{j}\right|
$$

The size of a sphere in the space of permutations with this distance is

$$
V(d, n)=\left|T_{d, n}\right|,
$$

where

$$
T_{d, n}=\left\{p \in S_{n}|| p_{i}-i \mid \leqslant d \text { for } 1 \leqslant i \leqslant n\right\} .
$$

For fixed $d$ it is well known that $V(d, n)$ satisfies a linear recurrence and that the generating function is a rational function (see Lehmer [5], Stanley [6]). Lehmer's proof

${ }^{*}$ The research was supported by the Norwegian Research Council 
was based on writing $V(d, n)$ as a permanent of a suitable matrix. He only considered $d \leqslant 3$. Stanley's proof is general and uses the transfer-matrix method, see $[6,4.7 .7]$. In [3] we studied $V(d, n)$ for general $d$, using permanent methods.

In the present paper we introduce two related new transfer-matrix methods. The advantage is that the underlying matrix has a small size.

\section{First transfer-matrix method.}

Let

$$
X=\left\{\left(x_{1}, x_{2}, \ldots, x_{d}\right) \mid d \geqslant x_{1} \geqslant x_{2} \geqslant \cdots \geqslant x_{d} \geqslant 0\right\} .
$$

It easy to see that $|X|=\left(\begin{array}{c}2 d \\ d\end{array}\right)$.

For $1 \leqslant j \leqslant d+1$ and $\mathbf{x} \in X$ we define

$$
\mathbf{x}^{j}=\left(x_{1}+1, x_{2}+1, \ldots, x_{j-1}+1, x_{j+1}, x_{j+2}, \ldots, x_{d}, 0\right) .
$$

In particular,

$$
\mathbf{x}^{1}=\left(x_{2}, x_{3}, \ldots, x_{d}, 0\right) \text { and } \mathbf{x}^{d+1}=\left(x_{1}+1, x_{2}+1, \ldots, x_{d}+1\right) .
$$

Let $T$ be the $|X| \times|X|$ transfer matrix where the rows and columns are indexed by $X$, and where

$$
\begin{aligned}
& \text { if } x_{1}<d, \text { then }\left\{\begin{array}{l}
t_{\mathbf{x}, \mathbf{x}^{j}}=1 \\
t_{\mathbf{x}, \mathbf{y}}=0 \\
\text { if } x_{1}=d \text {, then } j=1,2, \ldots, d+1 \\
t_{\mathbf{x}, \mathbf{x}^{1}}=1 \\
t_{\mathbf{x}, \mathbf{y}}=0
\end{array}\right. \text { otherwise, } \\
& \text { otherwise, }
\end{aligned}
$$

Theorem 1. For $d \geqslant 1, V(d, n)$ has generating function

$$
\sum_{n=0}^{\infty} V(d, n) z^{n}=\frac{\operatorname{det}(K)}{\operatorname{det}(I-z T)}=\frac{f_{d}(z)}{g_{d}(z)},
$$

where $K$ denotes the matrix obtained by removing the first row (row $\mathbf{0}$ ) and the first column (column $\mathbf{0}$ ) of $(I-z T)$, and

$$
\operatorname{gcd}\left(f_{d}(z), g_{d}(z)\right)=1 .
$$

Example 1. For $d=1$, the transfer matrix is

\begin{tabular}{c|ccc}
$\mathbf{x}$ & $\mathbf{y}:$ & $(0)$ & $(1)$ \\
\hline$(0)$ & & 1 & 1 \\
$(1)$ & & 1 & 0
\end{tabular}

Hence

$$
\sum_{n=0}^{\infty} V(1, n) z^{n}=\frac{\operatorname{det}(1)}{\operatorname{det}\left[\begin{array}{cc}
1-z & -z \\
-z & 1
\end{array}\right]}=\frac{1}{1-z-z^{2}}
$$


We recover equation (37) in Example 4.7.7 in Stanley [6], in which the underlying matrix is of dimension $7 \times 7$. Our matrix $T$ is of dimension $2 \times 2$.

Example 2. For $d=2$, our transfer matrix is

\begin{tabular}{l|ccccccc}
$\mathbf{x}$ & $\mathbf{y}:$ & $(00)$ & $(10)$ & $(11)$ & $(20)$ & $(21)$ & $(22)$ \\
\hline$(00)$ & & 1 & 1 & 1 & 0 & 0 & 0 \\
$(10)$ & 1 & 0 & 0 & 1 & 1 & 0 \\
$(11)$ & & 0 & 1 & 0 & 1 & 0 & 1 \\
$(20)$ & 1 & 0 & 0 & 0 & 0 & 0 \\
$(21)$ & & 0 & 1 & 0 & 0 & 0 & 0 \\
$(22)$ & & 0 & 0 & 0 & 1 & 0 & 0
\end{tabular}

Hence

$$
\begin{aligned}
& \operatorname{det}\left[\begin{array}{ccccc}
1 & 0 & -z & -z & 0 \\
-z & 1 & -z & 0 & -z \\
0 & 0 & 1 & 0 & 0 \\
-z & 0 & 0 & 1 & 0 \\
0 & 0 & -z & 0 & 1
\end{array}\right] \\
& \operatorname{det}\left[\begin{array}{cccccc}
1-z & -z & -z & 0 & 0 & 0 \\
-z & 1 & 0 & -z & -z & 0 \\
0 & -z & 1 & -z & 0 & -z \\
-z & 0 & 0 & 1 & 0 & 0 \\
0 & -z & 0 & 0 & 1 & 0 \\
0 & 0 & 0 & -z & 0 & 1
\end{array}\right] \\
&=\frac{1-z z^{2}}{1-z-2 z^{2}-2 z^{3}-2 z^{4}+z^{5}+z^{6}} \\
&=\frac{1-z}{1-2 z-2 z^{3}+z^{5}} .
\end{aligned}
$$

We recover the equation just before Example 4.7.17 in Stanley [6].

We now give a proof of Theorem 1.

Proof. For $\mathbf{x} \in X$, let $A_{\mathbf{x}}$ be the infinite matrix $\left(a_{i, j}\right)$ be defined by

$$
\begin{aligned}
& a_{i, j}=0 \text { for } j>i+d \text { or } i>j+d, \\
& a_{i, j}=0 \text { for } 1 \leqslant j \leqslant d \text { and } j+d-x_{j}<i \leqslant j+d, \\
& a_{i, j}=1 \text { otherwise. }
\end{aligned}
$$

Let $D$ be the directed graph whose vertices are $\left\{A_{\mathbf{x}} \mid \mathbf{x} \in X\right\}$. The arcs in $D$ are $\left(A_{\mathbf{x}}, A_{\mathbf{y}}\right)$ where the matrix $A_{\mathbf{y}}$ can be obtained by removing the first row and the $j$ 'th column $(j=1,2, \ldots, d+1)$ of $A_{\mathbf{x}}$. By the definition of $T$ we see that the adjacency matrix of $D$ is exactly T. By Stanley [6, Theorem 4.7.2], the right hand side of (1) is

$$
\frac{\operatorname{det}(K)}{\operatorname{det}(I-z T)}=\sum_{n=0}^{\infty} v(n) z^{n}
$$


where $v(n)$ is the number of closed walks of length $n$ based at $A_{\mathbf{0}}$. We claim that each such walk is in bijection with a permutation in $T_{d, n}$ so that $v(n)=V(d, n)$.

Referring to the original matrix $A_{\mathbf{0}}$, in the $i$ 'th step of the walk we remove row number $i$ and some column, column number $p_{i}$ say, where

$$
i-d \leqslant p_{i} \leqslant i+d .
$$

When the walk of length $n$ is closed, we have removed the first $n$ rows and $n$ column. Since we are left with (a new) $A_{0}$, the removed columns must be exactly the $n$ first. This also implies that $\left(p_{1}, p_{2}, \ldots, p_{n}\right)$ must be a permutation in $T_{d, n}$.

On the other hand, let $p=\left(p_{1} p_{2} \ldots p_{n}\right) \in T_{d, n}$. Define $h=\left(h_{1}, h_{2}, \ldots, h_{n}\right)$ by

$$
h_{i}=p_{i}-\left|\left\{j<i \mid p_{j}<p_{i}\right\}\right| .
$$

Since

$$
\left|\left\{j<i \mid p_{j}<p_{i}\right\}\right| \leqslant\left|\left\{j \in[n] \mid p_{j}<p_{i}\right\}\right|=p_{i}-1
$$

we have $h_{i} \geqslant 1$. Further, if $j \leqslant p_{i}-d-1$, then $p_{j} \leqslant j+d<p_{i}$. Hence

$$
\left|\left\{j<i \mid p_{j}<p_{i}\right\}\right| \geqslant p_{i}-d-1
$$

and so $h_{i} \leqslant d+1$. Therefore, $A_{\mathbf{z}^{h_{i}}}$ is well defined for all $i$ and all $\mathbf{z} \in X$.

We will show that the walk corresponding to $p$ is

$$
A_{\mathbf{0}} A_{\mathbf{0}^{h_{1}}} A_{\mathbf{0}^{h_{1} h_{2}}} \cdots A_{\mathbf{0}^{h_{1} h_{2} \cdots h_{n-1}}} A_{\mathbf{0}^{h_{1} h_{2} \cdots h_{n-1} h_{n}}} .
$$

Since $p$ is a permutation in $T_{d, n}$ we see by the argument above that

$$
A_{\mathbf{0}^{h_{1} h_{2} \cdots h_{n-1} h_{n}}}=A_{\mathbf{0}} .
$$

Moreover, we note that at the start of the $i$ 'th step, $\left|\left\{j<i \mid p_{j}<p_{i}\right\}\right|$ columns to the left of the column $p_{i}$ in the original $A_{\mathbf{0}}$ have already been removed. Therefore, at the $i$ 'th step, when we remove column $h_{i}$ in $A_{\mathbf{0}^{h_{1} h_{2} \cdots h_{i-1}}}$, this is exactly column

$$
\left|\left\{j<i \mid p_{j}<p_{i}\right\}\right|+h_{i}=p_{i}
$$

in the original $A_{\mathbf{0}}$. Hence, we see that the walk corresponds exactly to the permutation $p$.

It may be easier to understand the proof with diagrams, and we illustrate with an example below.

Example 3. A permutation $p \in S_{n}$ can be represented by the $n \times n$ matrix $B=\left(b_{i, j}\right)$ where $b_{i, p_{i}}=1$ and $b_{i, j}=0$ otherwise.

For example, consider $p=3142 \in T_{2,4}$. Then

$$
B=\left[\begin{array}{l}
0010 \\
1000 \\
0001 \\
0100
\end{array}\right] \text {. }
$$


Walk illustrated by removing rows/columns:

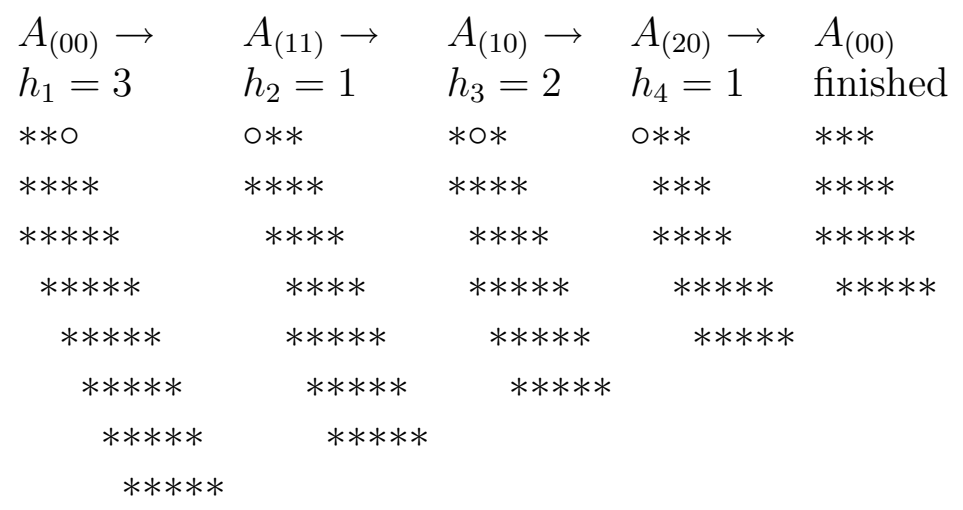

Walk illustrated by erasing rows/columns:

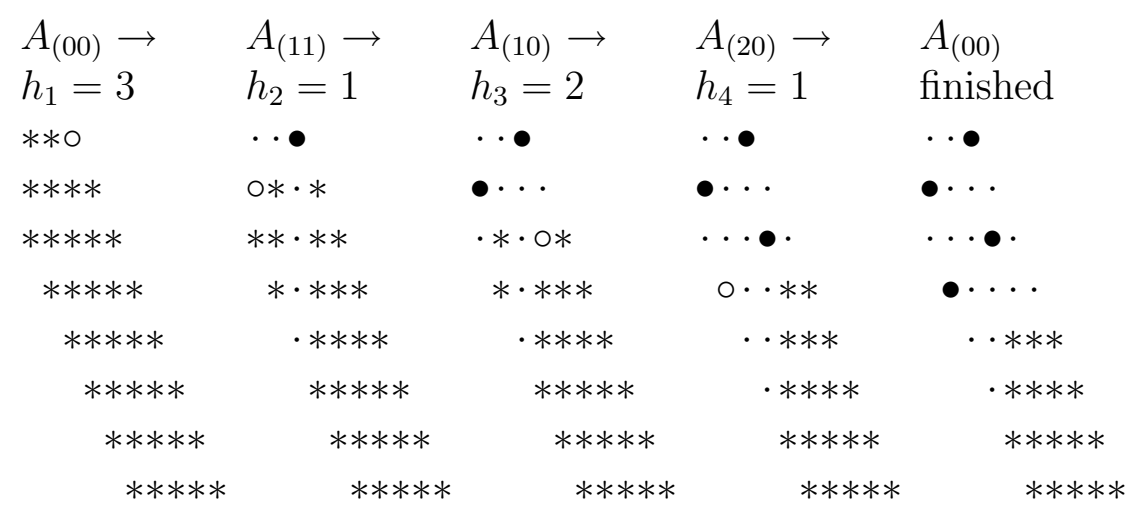

We can easily identify the matrix B in the last diagram.

Figure 1: Diagrams illustrating the first transfer-atrix method

Further, we get $h=(3,1,2,1)$. We have (by the definition of $\mathbf{x}^{j}$ )

$$
(00)^{3}=(11),(11)^{1}=(10),(10)^{2}=(20),(20)^{1}=(00) .
$$

Therefore, the closed walk corresponding to $p$ is

$$
A_{(00)} A_{(11)} A_{(10)} A_{(20)} A_{(00)} .
$$

The first diagram (in Fig. 1) shows the walk by using the "remove"-process, that is, removing the first row and column $h_{i}$ in the $i$ 'th step).

We write "*" for " 1 ", blank for " 0 ", and mark the column (and row) to be removed by "o".

The second diagram (in Fig. 1) shows the walk by using an "erase"-process (instead of removing the first row and the $h_{i}$ 'th column in the $i$ 'th step, we just erase these elements by changing "*" to "." to show the history of the process, moreover, "o" from previous steps are marked by "•"). 


\section{Second transfer-matrix method.}

For $1 \leqslant a \leqslant d+1$ and $1 \leqslant b \leqslant d$, let

$$
X_{a, b}=\left\{\left(x_{1}, x_{2}, \ldots, x_{d}\right) \mid a=x_{1} \geqslant x_{2} \geqslant \cdots \geqslant x_{b}>0 \text { and } x_{i}=0 \text { for } i>b\right\} .
$$

For $0 \leqslant a \leqslant d$ and $0 \leqslant b \leqslant d$, let

$$
Y_{a, b}=\left\{\left(x_{1}, x_{2}, \ldots, x_{d}\right) \mid a=x_{1} \geqslant x_{2} \geqslant \cdots \geqslant x_{b} \geqslant 0 \text { and } x_{i}=0 \text { for } i>b\right\}
$$

Let

$$
Y=\bigcup_{a=0}^{d-1} Y_{a, d-a} .
$$

For $\mathbf{y}=\left(y_{1}, y_{2}, \ldots, y_{b}, 0,0, \ldots, 0\right) \in X_{a, b}$, let

$$
\mathbf{y}^{-}=\left(y_{2}-1, y_{3}-1, \ldots, y_{b}-1,0,0, \ldots, 0\right) \in Y_{y_{2}-1, b-1} .
$$

For a pair $\mathbf{x}, \mathbf{y} \in X$, let $A_{\mathbf{x}, \mathbf{y}}$ be the infinite matrix $\left(a_{i, j}\right)$ be defined by

$$
\begin{aligned}
& a_{i, j}=0 \text { for } j>i+d \text { or } i>j+d, \\
& a_{i, j}=0 \text { for } 1 \leqslant i \leqslant d \text { and } i+d-x_{i}<j \leqslant i+d \\
& a_{i, j}=0 \text { for } 1 \leqslant j \leqslant d \text { and } j+d-y_{j}<i \leqslant j+d \\
& a_{i, j}=1 \text { otherwise, }
\end{aligned}
$$

We note that in the first row of this matrix, the first $d+1-x_{1}$ elements are 1 , the remaining are 0 .

Let $\leqslant$ denote the lexicographic ordering, that is

$$
\mathbf{y} \leqslant \mathbf{x} \text { if } \mathbf{y}=\mathbf{x} \text { or } y_{i}=x_{i} \text { for } 1 \leqslant i<j \text { and } y_{j}<x_{j} \text { for some } j \text {. }
$$

We define three classes of pairs of sequences:

$$
\begin{aligned}
& Z_{1}=\{(\mathbf{x}, \mathbf{0}) \mid \mathbf{x} \in Y\}, \\
& Z_{2}=\left\{(\mathbf{x}, \mathbf{y}) \mid \mathbf{x} \in Y_{a, d-a}, \mathbf{y} \in Y_{b, d-a}, \text { where } 1 \leqslant b \leqslant a \leqslant d-1 \text { and } \mathbf{y} \leqslant \mathbf{x}\right\} \\
& Z_{3}=\left\{\left(\mathbf{x}, \mathbf{y}^{-}\right) \mid \mathbf{x}, \mathbf{y} \in X_{a, d+1-a}, \text { where } 1 \leqslant a \leqslant d \text { and } \mathbf{x} \leqslant \mathbf{y}\right\} .
\end{aligned}
$$

Let $Z=Z_{1} \cup Z_{2} \cup Z_{3}$. A relatively simple calculation shows that

$$
|Z|=\frac{1}{2}\left(\begin{array}{c}
2 d \\
d
\end{array}\right)+2^{d-1}
$$

For $\mathbf{x}, \mathbf{z} \in Y$, where $\mathbf{x} \neq \mathbf{z}$, define $U_{\{\mathbf{x}, \mathbf{z}\}}=\left\{A_{\mathbf{x}, \mathbf{z}}, A_{\mathbf{z}, \mathbf{x}}\right\}$. The set of vertices is defined by

$$
\mathcal{M}_{2}=\left\{U_{\{\mathbf{x}, \mathbf{z}\}} \mid(\mathbf{x}, \mathbf{z}) \in Z\right\} \text {. }
$$


Remark. We have $\mathbf{z} \leqslant \mathbf{x}$ for any pair $(\mathbf{x}, \mathbf{z}) \in Z$. Hence, given $U_{\{\mathbf{u}, \mathbf{v}\}} \in \mathcal{M}_{2}$, we can uniquely determine if $(\mathbf{u}, \mathbf{v}) \in Z$ or $(\mathbf{v}, \mathbf{u}) \in Z$.

Consider $U_{\{\mathbf{x}, \mathbf{z}\}} \in \mathcal{M}_{2}$ where $(\mathbf{x}, \mathbf{z}) \in Z$. For $1 \leqslant j \leqslant d+1-x_{1}$ there is an arc from $U_{\{\mathbf{x}, \mathbf{z}\}}$ to $U_{\left\{\mathbf{x}^{\prime}, \mathbf{z}^{\prime}\right\}}$, where

$$
\mathbf{x}^{\prime}=\left(x_{2}, x_{3}, \ldots, x_{d}, 0\right)
$$

and

$$
\mathbf{z}^{\prime}=\left(z_{1}+1, z_{2}+1, \ldots, z_{j-1}+1, z_{j+1}, z_{j+2}, \ldots, z_{d}, 0\right) .
$$

This is well defined since for the matrix $A_{\mathbf{x}, \mathbf{z}}$,

$$
a_{i, d+1-x_{1}}=1 \text { for } 1 \leqslant i \leqslant 2 d+1-x_{1},
$$

that is, there are no "extra" zeros in column $d+1-x_{1}$. Moreover, the set of extra zeros determined by $\mathbf{x}$ and the set of extra zeros determined by $\mathbf{z}$ are disjoint. We must show that $U_{\left\{\mathbf{x}^{\prime}, \mathbf{z}^{\prime}\right\}} \in \mathcal{M}_{2}$, that is $\left(\mathbf{x}^{\prime}, \mathbf{z}^{\prime}\right) \in Z$ or $\left(\mathbf{z}^{\prime}, \mathbf{x}^{\prime}\right) \in Z$. We split the proof into cases.

Case I) $(\mathbf{x}, \mathbf{z}) \in Z_{1}$ (where $(\mathbf{z}=\mathbf{0})$. Then $\mathbf{x} \in X_{a, l}$ where $1 \leqslant l \leqslant d-a$.

Subcase I.a) $j=1$. Then $\mathbf{z}^{\prime}=\mathbf{0}$. Hence $\left(\mathbf{x}^{\prime}, \mathbf{z}^{\prime}\right) \in Z_{1}$.

Subcase I.b) $1<j \leqslant d+1$. Then $\mathbf{z}^{\prime}=(1,1, \ldots, 1,0,0, \ldots, 0) \in X_{1, j-1} \subset Y$.

Subsubcase I.b.1) $\mathbf{z}^{\prime}<\mathbf{x}^{\prime}$. Then $\left(\mathbf{x}^{\prime}, \mathbf{z}^{\prime}\right) \in Z_{2}$.

Subsubcase I.b.2) $\mathbf{x}^{\prime}=\mathbf{0}$. Then $\left(\mathbf{z}^{\prime}, \mathbf{x}^{\prime}\right) \in Z_{1}$.

Subsubcase I.b.3) $\mathbf{x}^{\prime}=(1,1, \ldots, 1,0,0, \ldots, 0) \in X_{1, i}$ where $i \leqslant j-1$. Then $\left(\mathbf{z}^{\prime}, \mathbf{x}^{\prime}\right) \in Z_{2}$.

Case II $)(\mathbf{x}, \mathbf{y}) \in Z_{2}$. Then $\mathbf{x} \in X_{a, l}$ and $\mathbf{y} \in X_{b, m}$ where

$$
1 \leqslant b \leqslant a, 1 \leqslant l \leqslant d-a, \text { and } 1 \leqslant m \leqslant d-a .
$$

In this case, we get $\mathbf{x}^{\prime} \in X_{x_{2}, d-1-a} \subset Y_{x_{2}, d-x_{2}}$ since

$$
d-1-a=d_{1}-x_{1} \leqslant d-1-x_{2}<d-x_{2} .
$$

Subcase II.a) $j=1$. Then $\mathbf{y}^{\prime}=\left(y_{2}, \ldots, y_{m}, 0, \ldots, 0\right) \in X_{y_{2}, m-1}$. If $\mathbf{y}^{\prime} \leqslant \mathbf{x}^{\prime}$, then $\left(\mathbf{x}^{\prime}, \mathbf{y}^{\prime}\right) \in Z_{2}$ since $X_{y_{2}, m-1} \subset Y_{y_{2}, d-x_{2}}$

$$
\text { (because } m-1 \leqslant d-a-1<d-x_{2} \text { ). }
$$

On the other hand, if $\mathbf{x}^{\prime} \leqslant \mathbf{y}^{\prime}$, then

$$
x_{2} \leqslant y_{2} \text { and } d-1-a \leqslant d-1-b \leqslant d-1-y_{2}<d-y_{2}
$$

and so $\mathbf{x}^{\prime} \in Y_{x_{2}, d-y_{2}}$ and $\left(\mathbf{y}^{\prime}, \mathbf{x}^{\prime}\right) \in Z_{2}$.

Subcase II.b) $1<j \leqslant m$. Then

$$
\mathbf{y}^{\prime}=\left(y_{1}+1, y_{2}+1, \ldots, y_{j-1}+1, y_{j+1}, \ldots, y_{m}, 0, \ldots, 0\right) \in X_{y_{1}+1, m-1} .
$$

If $\mathbf{y}^{\prime} \leqslant \mathbf{x}^{\prime}$, then $\left(\mathbf{x}^{\prime}, \mathbf{y}^{\prime}\right) \in Z_{2}$ since $X_{y_{1}+1, m-1} \subset Y_{y_{1}+1, d-x_{2}}$

$$
\text { (because } m-1 \leqslant d-a-1<d-x_{2} \text { ). }
$$


On the other hand, if $\mathbf{x}^{\prime} \leqslant \mathbf{y}^{\prime}$, we must have $x_{2} \leqslant y_{2}+1$ and so $d-x_{2} \leqslant d-y_{2}$, that is, $\mathbf{x}^{\prime} \in Y_{x_{2}, d-y_{2}}$. Hence then $\left(\mathbf{y}^{\prime}, \mathbf{x}^{\prime}\right) \in Z_{2}$.

Subcase II.c) $m+1 \leqslant j \leqslant d-a$. We get

$$
\mathbf{y}^{\prime}=\left(y_{1}+1, y_{2}+1, \ldots, y_{m}+1,1, \ldots, 1,0, \ldots, 0\right) \in X_{y_{1}+1, j-1} .
$$

We have $j-1 \leqslant d-a-1<d-x_{2}$. Hence if $\mathbf{y}^{\prime} \leqslant \mathbf{x}^{\prime}$, then $\left(\mathbf{x}^{\prime}, \mathbf{y}^{\prime}\right) \in Z_{2}$. On the other hand, if $\mathbf{x}^{\prime} \leqslant \mathbf{y}^{\prime}$, then $m-1 \leqslant d-a-1 \leqslant d-y_{1}-1$ and so $\mathbf{x}^{\prime} \in Y_{x_{2}, d-y_{1}-1}$. Hence $\left(\mathbf{y}^{\prime}, \mathbf{x}^{\prime}\right) \in Z_{2}$.

Subcase II.d) $j=d+1-a$. We get

$$
\mathbf{y}^{\prime}=\left(y_{1}+1, y_{2}+1, \ldots, y_{m}+1,1, \ldots, 1,0, \ldots, 0\right) \in X_{y_{1}+1, d-a} .
$$

Subsubcase II.d.1) $\mathbf{y}^{\prime} \leqslant \mathbf{x}^{\prime}$. Then $\left(\mathbf{x}^{\prime}, \mathbf{y}^{\prime}\right) \in Z_{2}$.

Subsubcase II.d.2) $\mathbf{x}^{\prime}<\mathbf{y}^{\prime}$ and $y_{1}+1 \leqslant a$. Then $\left(\mathbf{y}^{\prime}, \mathbf{x}^{\prime}\right) \in Z_{2}$.

Subsubcase II.d.3) $y_{1}+1=a+1$. Note that $x_{1}+1=a+1$. Let

$$
\mathbf{u}=\left(a+1, x_{2}+1, \ldots, x_{d-a}+1,0, \ldots, 0\right) .
$$

Then $\mathbf{u}^{-}=\mathbf{x}^{\prime}$. Since $\mathbf{y} \leqslant \mathbf{x}, \mathbf{y}^{\prime} \leqslant \mathbf{u}$. Hence $\left(\mathbf{y}^{\prime}, \mathbf{x}^{\prime}\right)=\left(\mathbf{y}^{\prime}, \mathbf{u}^{-}\right) \in Z_{3}$.

Case III $)(\mathbf{x}, \mathbf{z}) \in Z_{3}$ where $\mathbf{z}=\mathbf{y}^{-}, \mathbf{x}, \mathbf{y} \in X_{a, d+1-a}$ and $\mathbf{x} \leqslant \mathbf{y}$. In this case, we get $\mathbf{x}^{\prime} \in X_{x_{2}, d-a} \subset Y$. We have $\mathbf{z} \in X_{y_{2}-1, m}$ for some $m \leqslant d-a$.

Subcase III.a) $j=1$. If $\mathbf{z}^{\prime}=\mathbf{0}$, then $\left(\mathbf{x}^{\prime}, \mathbf{z}^{\prime}\right) \in Z_{1}$. Otherwise, $\left(\mathbf{x}^{\prime}, \mathbf{z}^{\prime}\right) \in Z_{2}$ or $\left(\mathbf{z}^{\prime}, \mathbf{x}^{\prime}\right) \in Z_{2}$. Subcase III.b) $j=1$. If $\mathbf{z}^{\prime}=\mathbf{0}$, then $\left(\mathbf{x}^{\prime}, \mathbf{z}^{\prime}\right) \in Z_{1}$. Otherwise, $\left(\mathbf{x}^{\prime}, \mathbf{z}^{\prime}\right) \in Z_{2}$ or $\left(\mathbf{z}^{\prime}, \mathbf{x}^{\prime}\right) \in Z_{2}$. Subcase III.c) $1<j \leqslant m$. Then

$$
\mathbf{z}^{\prime}=\left(y_{2}, y_{3}, \ldots, y_{j-1}, y_{j+1}-1, \ldots, y_{m}-1,0, \ldots, 0\right) .
$$

Again, $\left(\mathbf{x}^{\prime}, \mathbf{z}^{\prime}\right) \in Z_{2}$ or $\left(\mathbf{z}^{\prime}, \mathbf{x}^{\prime}\right) \in Z_{2}$.

Subcase III.d) $m+1 \leqslant j \leqslant d+1-a$. Then

$$
\mathbf{z}^{\prime}=\left(y_{2}, y_{3}, \ldots, y_{m}, 1, \ldots, 1,0, \ldots, 0\right) .
$$

Subsubcase III.d.1) $j \leqslant d-a$ or $y_{2}<a$. Then $\left(\mathbf{x}^{\prime}, \mathbf{z}^{\prime}\right) \in Z_{2}$ or $\left(\mathbf{z}^{\prime}, \mathbf{x}^{\prime}\right) \in Z_{2}$.

Subsubcase III.d.2) $j=d+1-a$ and $y_{2}=a$. Then $\mathbf{z} \in X_{a, d-a}$ and so $\left(\mathbf{x}^{\prime}, \mathbf{z}^{\prime}\right) \in Z_{2}$ or $\left(\mathbf{z}^{\prime}, \mathbf{x}^{\prime}\right) \in Z_{2}$ also in this case.

An $n$ step path from $U_{\{\mathbf{0}, \mathbf{0}\}}$ to $U_{\{\mathbf{0}, \mathbf{0}\}}$ will remove the first $n$ rows and the first $n$ columns of $A_{\mathbf{0 , 0}}$. Hence, it corresponds to a permutation.

To describe the path that corresponds to a given permutation $p \in T_{d, n}$ is similar to the first transfer-matrix method, but a little more involved. Let $p \in T_{d, n}$, let $B$ be the matrix corresponding to $p$, and let $q$ be the permutation corresponding to the transposed of $B$.

We start with $A_{\mathbf{0}, \mathbf{0}}$. Let $A_{\mathbf{x}, \mathbf{z}}$ be the matrix we have after $k-1$ steps. Let the first row of $A_{\mathbf{x}, \mathbf{z}}$ be row number $r$ and the first column of $A_{\mathbf{x}, \mathbf{z}}$ be column number $s$ of the original $A_{\mathbf{0}, \mathbf{0}}$. Let the number of erased columns $j$ such that $j<p_{r}$ be $t_{k}$ and the number 
of erased rows $i$ such that $i<q_{s}$ be $u_{k}$. Since $A_{\mathbf{0}, \mathbf{0}}$ has a 1 in position $\left(r, p_{r}\right), A_{\mathbf{x}, \mathbf{z}}$ must have a 1 in position $\left(1, p_{r}-t_{k}\right)$. Similarly, it has a 1 in position $\left(q_{s}-u_{k}, 1\right)$. In the $k^{\prime}$ th step, if $\mathbf{x} \leqslant \mathbf{z}$, then remove the first row of $A_{\mathbf{x}, \mathbf{z}}$ and column number $p_{r}-t_{k}$. Similarly, if $\mathbf{x}>\mathbf{z}$, then remove the first column and row number $q_{s}-u_{k}$. By this process, in each step we remove a row/column pair corresponding to a 1 in matrix $B$. Hence, the path corresponds exactly to the permutation $p$.

Example 4. For $d=2$, the vertices are

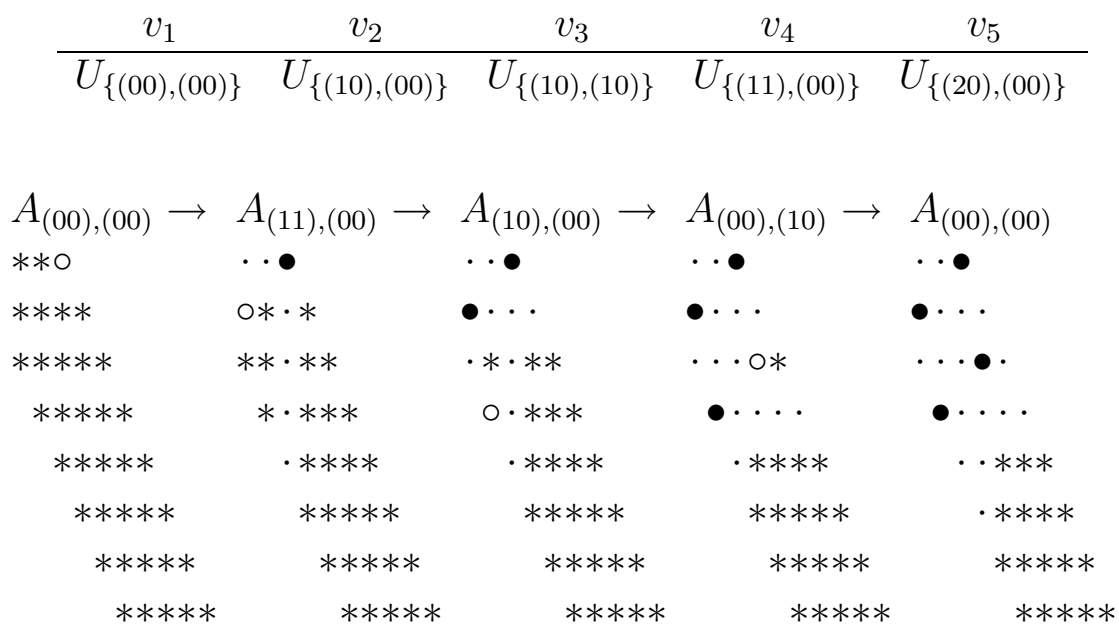

Figure 2: Walk in second method for the permutation (3142) illustrated by erasing rows/columns

The transfer matrix is

\begin{tabular}{c|ccccc} 
& $v_{1}$ & $v_{2}$ & $v_{3}$ & $v_{4}$ & $v_{5}$ \\
\hline$v_{1}$ & 1 & 1 & 0 & 1 & 0 \\
$v_{2}$ & 1 & 1 & 0 & 0 & 0 \\
$v_{3}$ & 1 & 0 & 0 & 0 & 1 \\
$v_{4}$ & 0 & 1 & 1 & 0 & 0 \\
$v_{5}$ & 1 & 0 & 0 & 0 & 0
\end{tabular}

Hence

$$
\frac{\operatorname{det}(K)}{\operatorname{det}(I-z T)}=\frac{\operatorname{det}\left[\begin{array}{cccc}
1-z & 0 & 0 & 0 \\
0 & 1 & 0 & -z \\
-z & -z & 1 & 0 \\
0 & 0 & 0 & 1
\end{array}\right]}{\operatorname{det}\left[\begin{array}{ccccc}
1-z & -z & 0 & -z & 0 \\
-z & 1-z & 0 & 0 & 0 \\
-z & 0 & 1 & 0 & -z \\
0 & -z & -z & 1 & 0 \\
-z & 0 & 0 & 0 & 1
\end{array}\right]}=\frac{1-z}{1-2 z-2 z^{3}+z^{5}}
$$


as before, but now with a $5 \times 5$ matrix. Also $\operatorname{gcd}(\operatorname{det}(K), \operatorname{det}(I-T z))=1$ in this case. The diagram in Fig. 2 shows the walk for the permutation (3142) by using the "erase"-process for this graph.

\section{On $\operatorname{deg} f_{d}(z)$ and $\operatorname{deg} g_{d}(z)$.}

In Theorem 1 we showed that the generating function for $V(d, n)$ is a rational function $f_{d}(z) / g_{d}(z)$.

Theorem 2. For all $d \geqslant 1$ we have

$$
\operatorname{deg} f_{d}(z) \leqslant \operatorname{deg} g_{d}(z)-2 d .
$$

Proof. Consider the matrix $K$ in Theorem 1. Since $t_{(d 00 \ldots 0), \mathbf{y}}=1$ only for $\mathbf{y}=(000 \ldots 0)$, the row $(d 00 \ldots 0)$ in $K$ contains a single 1 (in column $(d 00 \ldots 0)$ ) and zeros otherwise. Hence we can remove row and column $(d 00 \ldots 0)$ in $K$ without changing the value of $\operatorname{det}(K)$. Similarly, $t_{(d d 00 \ldots 0), \mathbf{y}}=1$ only for $\mathbf{y}=(d 00 \ldots 0)$. Hence, the reduced matrix $K$ contains a single 1 in row $(d d 00 \ldots 0)$ and so row and column $(d d 00 \ldots 0)$ in $K$ can also be removed without changing the value of $\operatorname{det}(K)$. The same argument and induction shows that we can remove all $d$ rows and columns $(d d \ldots d 00 \ldots 0)$.

Column $(111 \ldots 1)$ contains a single 1 since $t_{\mathbf{x},(111 \ldots 1)}=1$ only for $\mathbf{x}=(000 \ldots 0)$. Hence row and column $(111 \ldots 1)$ can also be removed without changing the value of $\operatorname{det}(K)$. In general, for $1 \leqslant r \leqslant d, t_{\mathbf{x},(r r r \ldots r)}=1$ only for $\mathbf{x}=(r-1, r-1, r-1, \ldots, r-1)$. Hence, induction shows that all rows and columns $(r r r \ldots r)$ can also be removed without changing the value of $\operatorname{det}(K)$ for $r=1,2, \ldots, d-1$ (note that $(d d d \ldots d)$ has already been removed). In all we can remove $2 d-1 \mathrm{row} /$ column pairs. The reduced matrix with the same determinant as $K$ has dimension $2 r$ less than the dimension of $T$.

The second transfer-matrix method shows that

$$
\operatorname{deg} g_{d}(z) \leqslant|Z|=2^{d-1}+\frac{1}{2}\left(\begin{array}{c}
2 d \\
d
\end{array}\right) .
$$

We have computed the generating functions for $d \leqslant 6$. They are listed in the appendix of [3]. For these examples, we have equality in both (3) and (2). This limited evidence indicate that we may have equality in both (3) and (2) in general. In particular, this would imply that

$$
\operatorname{gcd}(\operatorname{det}(K)), \operatorname{det}(I-z T))=1
$$

for the second transfer-matrix method, and that the matrix in the second transfer-matrix method is smallest possible for any transfer-matrix for this problem. 


\section{Acknowledgement}

My original proofs in [3] were based on expanding a permanent. It was pointed out by one of the referees that the transfer-matrix method gives a more direct and elegant proof of the generating function. Moreover it also gives a more explicit expression for $\sum V(d, n) z^{n}$. They even gave a sketch of a presentation of the first transfer-matrix method. Their input has greatly improved this paper.

\section{References}

[1] A. Jiang, R. Mateescu, M. Schwartz and J. Bruck, "Rank modulation for flash memories," in Proc. IEEE Internat. Symp. on Inform. Th., 2008, pp. 1731-1735.

[2] A. Jiang, M. Schwartz and J. Bruck, "Error-correcting codes for rank modulation," in Proc. IEEE Internat. Symp. on Inform. Th., 2008, pp. 1736-1740.

[3] T. Kløve, "Spheres of permutations under the infinity norm - Permutations with limited displacement," Reports in Informatics, Dept. of Informatics, Univ. Bergen, Report no. 376, November 2008.

Online: http://www.ii.uib.no/publikasjoner/texrap/pdf/2008-376.pdf

[4] T. Kløve, T.-T. Lin, S.-C. Tsai, W.-G. Tzeng, "Permutation arrays under the Chebyshev distance." http://arxiv.org/abs/0907.2682

[5] D. H. Lehmer, "Permutations with strongly restricted displacements," in Combinatorial Theory and its Applications II, P. Erdős, A. Rényi and V. T. Sós (eds.), Amsterdam: North Holland Publ., 1970, pp. 755-770.

[6] R. P. Stanley, Enumerative Combinatorics, Vol. I. Cambridge, U.K.: Cambridge Univ. Press, 1997. 